\title{
Catégorisations et représentations sociolinguistiques : les variétés stylistiques existent-elles?
}

\author{
Buson Laurence (a), Chevrot Jean-Pierre (b) \& Nardy Aurélie (c), Abouzaïd Myriam (d) \\ (a) Laurence.Buson@u-grenoble3.fr Université Grenoble-Alpes - Laboratoire Lidilem \\ (b) Jean-Pierre.Chevrot@u-grenoble3.fr Université Grenoble-Alpes - Laboratoire Lidilem - IUF \\ (c) Aurelie.Nardy@u-grenoble3.fr Université Grenoble-Alpes - Laboratoire Lidilem \\ (d) Myriam.Abouzaid@u-grenoble3.fr Université Grenoble-Alpes - Laboratoire Lidilem
}

\section{Introduction}

Dans le sens commun, les langues sont perçues comme des entités homogènes dont la connaissance est partagée par l'ensemble des locuteurs qui les parlent. Malgré cette perception globalisante, l'observation de l'usage quotidien du langage amène à distinguer différentes façons de parler la même langue. Ces variétés sont associées à des situations ou à des intentions particulières (parler « familier »), ainsi qu'à des positions dans l'espace social ou géographique (parler « populaire », " avec l'accent du midi »). Depuis quatre décennies, la sociolinguistique a conceptualisé cette hétérogénéité fondamentale des langues sous la forme de variables sociolinguistiques, qui permettent au locuteur de dire la même chose avec des variantes de valeur sociale et stylistique différentes (Labov, 1972). Par exemple, la réalisation optionnelle du premier élément de la négation en français (je (ne) viens pas demain) (Armstrong, 2002) ou le fait de prononcer ou non certaines liaisons (De Jong, 1994) sont des variables sociolinguistiques bien décrites du français.

Les enquêtes sociolinguistiques menées dans de nombreuses langues ont montré que ces points de variation étaient sujets au jugement social. Les locuteurs impliqués dans les tâches de jugement associent les variantes standard (par exemple maintenir le ne de la négation) au prestige social, au niveau d'études, à l'ambition professionnelle alors que les variantes non standard sont liées à la sociabilité, à la rudesse et à la loyauté envers le groupe d'origine (Lafontaine, 1986; Trudgill, 1975). En outre, la fréquence des différents types de variantes dans les énoncés des locuteurs dépend simultanément de leurs caractéristiques sociodémographiques (genre, classe sociale, âge) et du contexte de l'échange (Coupland, 2007 ; Labov, 1972, 2001; Trudgill, 1995 inter alia). C'est cette dernière dimension qui définit différents styles sociolinguistiques reliés à des paramètres contextuels de granularité variable. Ainsi, on a pu montrer que la fréquence des variantes chez un locuteur dépendait d'oppositions globales entre des situations dites formelles (par exemple des interactions maitre-élève(s) ou des entretiens d'embauche) et informelles (par exemple des conversations entre amis) ainsi que de paramètres plus locaux de l'interaction (thème de la conversation, changement d'interlocuteur, recherche de connivence, etc.).

La perception du style et les fonctionnements cognitifs de catégorisation qui permettent au locuteur de classer les énoncés qu'il entend en différentes variétés discrètes (par exemple, styles « soutenu », « courant », "familier ») ont fait l'objet de peu de recherches (Eckert \& Rickford, 2001; Coupland, 2007 ; Gadet, 2007), sans doute notamment du fait de l'émergence encore très récente de la dimension cognitive dans le champ de la sociolinguistique (Campbell-Kibler, 2010 ; Loudermilk, 2013 ; Chevrot \& Foulkes, 2013). Cette perspective ouvre pourtant des possibilités nouvelles pour comprendre l'interaction entre les phénomènes de catégorisation et les aspects sociaux et culturels, une problématique cruciale (Lahire \& Rosental, 2008), que nous traitons ici en mobilisant la sociolinguistique et la psychologie sociale. En outre, cette problématique interroge, avec une focale et des méthodes nouvelles, des questions récurrentes en sociolinguistique concernant la cohérence du style, les stéréotypes et la saillance des traits linguistiques en réception des messages. 
Les énoncés hétérogènes sur le plan stylistique (par exemple, un mélange de traits soutenus et familiers) sont pourtant considérés par les locuteurs d'une langue comme des ensembles homogènes qu'ils catégorisent en les nommant (Gadet, 2007). Cette catégorisation pose plusieurs questions en ce qui concerne les dispositifs cognitifs qui sous-tendent le traitement et les représentations du style. Premièrement, certains traits linguistiques particulièrement saillants pourraient être perçus prioritairement par les locuteurs (Landragin, 2004, 2005) et permettre, par un effet de halo (Moreau \& Brichard, 1997), de mobiliser une représentation cohérente du style qui aboutirait à requalifier l'ensemble de l'énoncé en omettant les éléments linguistiques dissonants. Deuxièmement, on sait que diverses informations sociales modifient la perception même des variantes sociolinguistiques : voix évoquant un genre, photo mettant en évidence le groupe social du locuteur, évocation incidente d'une aire géographique par un emblème (Niedzielski, 1999; Johnson et al., 1999; Hay et al., 2006a, 2006b ; Hay \& Drager, 2010 ; Staum Casasanto, 2009; Squires, 2011). Les stéréotypes et les idéologies linguistiques pourraient donc influencer les processus qui aboutissent à la catégorisation des énoncés. Troisièmement, cette catégorisation pourrait également résulter de l'interaction entre les modalités ascendantes et les modalités descendantes que nous venons de décrire.

À la suite d'une première étude exploratoire où nous demandions à des enfants de 9 à 11 ans de réagir à l'audition d'énoncés différents sur le plan stylistique (Buson, 2010), un modèle de perception des variétés stylistiques a pu être avancé, dont nous synthétisons les caractéristiques principales dans le schéma ciaprès.

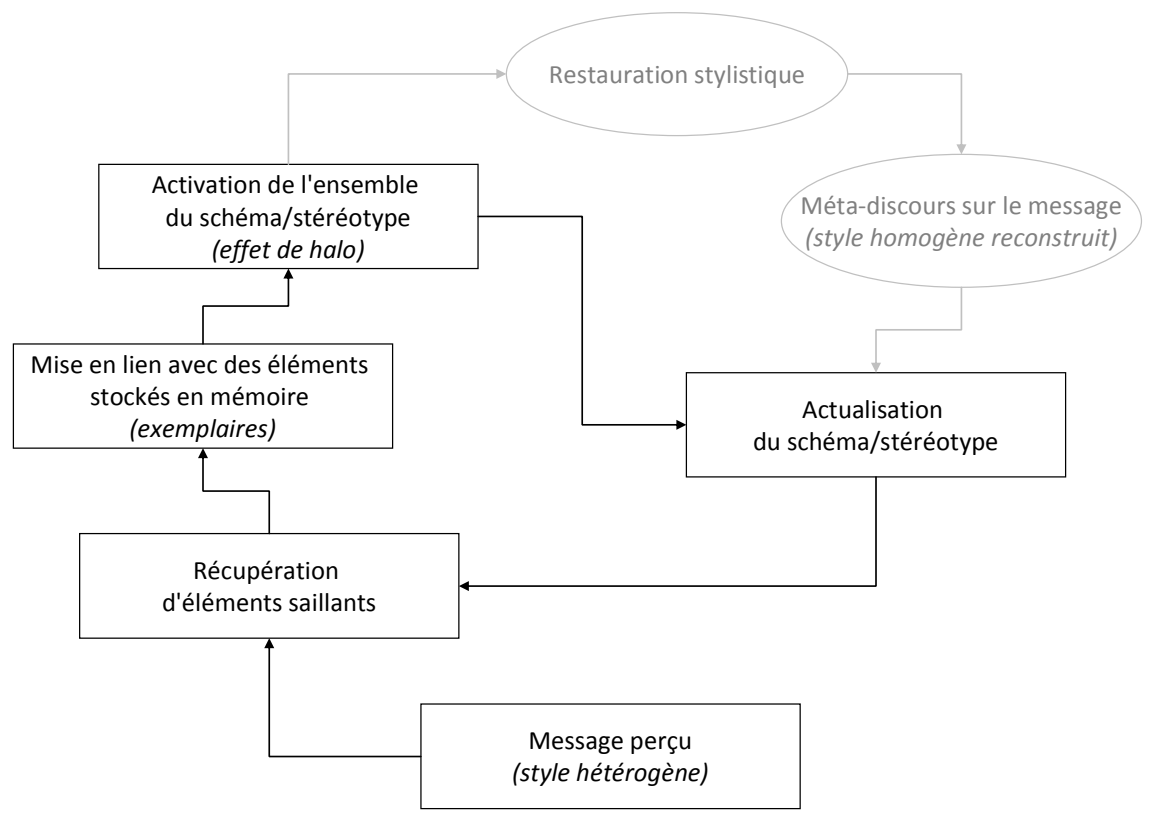

Figure 1 : processus de perception des variétés stylistiques

Selon cette première étude exploratoire, la perception du style serait un processus en hélice s'alimentant des modalités perceptives descendantes (stéréotype) et ascendantes (saillance); le récepteur s'appuierait sur un ou plusieurs éléments stylistiquement saillants de l'énoncé pour activer une catégorie globale/un stéréotype (ex : langage «soutenu », langage "familier »). Cette activation du stéréotype a été mise en évidence grâce à un phénomène que nous avons nommé restauration stylistique ${ }^{1}$ (désormais RS) : nous avons en effet observé, de manière incidente, que, quand le sujet répète un énoncé, il ajoute des éléments absents de l'input qu'il vient d'entendre mais cohérents avec le schéma/stéréotype qu'il a en mémoire. Les exemples suivants illustrent ce phénomène (pour davantage de détails, se reporter à Buson, 2010). 


\section{Énoncé entendu :}

bonjour - je suis désolé je suis momentanémenT (liaison /t/) absent - mais si vous le souhaitez vous pouvez $\mathrm{m}(\mathrm{e}$ ) laisseR (liaison /R/) un message avec vos coordonnées et $\mathrm{j}(\mathrm{e})$ vous recontacterai ultérieurement - je vous remercie et à bientôt

Énoncé repris par l'enfant lors de son commentaire de l'énoncé entendu :

Exemple 1: «la première il dit pas des choses comme ça, il dit "désolé je ne suis pas là" » (Margaux, 9 ans)

Exemple 2 : «le premier c'était quand il dit "vous pouvez me laisser un message car je ne suis pas là" » (Seynabou, 10 ans)

Nous constatons que l'énoncé entendu, plutôt formel (lexique surveillé, liaisons facultatives réalisées, surveillance articulatoire), est repris dans les commentaires des enfants, non pas à la lettre mais à l'esprit, et qu'ils produisent des marques stéréotypiques des styles formels tels que des ne de négation, pourtant absents du message entendu.

L'existence de ce processus de RS, central dans la mise en évidence des liens entre saillance et stéréotype, permettrait de confirmer l'existence de catégorisations cognitives du style en variétés dénombrables et discrètes, pouvant expliquer la difficulté des locuteurs à concevoir l'hétérogénéité des registres. Alors que les linguistes déconstruisent la notion de style en constatant son caractère non homogène, non fini et non dénombrable et proposent de le concevoir sous forme de continuum multidimensionnel (Gadet, 2007), les représentations des locuteurs - contrairement à leurs productions effectives - pourraient s'organiser en catégories. La mise en évidence de ce hiatus entre les productions linguistiques et les représentations de ces productions est intéressante pour clarifier la compréhension du lien entre émission et réception des messages. La validation expérimentale du modèle (cf. schéma ci-dessus) pourrait notamment expliquer les effets de "gommage» (erasure, cf. Irvine, 2001) dans la réception des énoncés : si le ou les traits dissonants dans le message ne sont pas suffisamment saillants, ils ne sont pas repérés ni pris en compte dans le processus de catégorisation.

Nous avons donc cherché à mettre en évidence l'existence de ce phénomène de manière plus systématique, auprès de locuteurs adultes natifs du français.

\section{Méthodologie de l'étude}

Afin de systématiser l'observation du phénomène de RS, nous avons mis en place une expérimentation auprès de 58 adultes âgés de 18 à 25 ans, 25 hommes et 33 femmes, tous francophones natifs.

Ces sujets ont effectué une tâche de répétition d'énoncés formels ou informels, stylistiquement homogènes ou non homogènes. Les énoncés stylistiquement homogènes incluent uniquement des variantes sociologuistiques cohérentes, compatibles avec leur contenu et les choix lexicaux. Par exemple, dans un énoncé formel homogène, toutes les liaisons sont réalisées ainsi que les ne de négation, le lexique est académique (certes, hémicycle) et le contenu renvoie à des débats de société ou à des généralités (la vie politique ou l'éducation). Un énoncé formel non homogène partage tous ces traits à l'exception d'une seule variante qui les contredit: une liaison n'est pas réalisée, le ne de la négation est omis, etc. L'ensemble du matériel linguistique est constitué de 24 énoncés à répéter ( $c f$. annexe 1$)$ : 6 énoncés formels homogènes (codés FH dans les graphiques), 6 énoncés formels non homogènes (codés FnH), 6 énoncés informels homogènes (codés $\mathrm{IH}$ ), 6 énoncés informels non homogènes (codés $\mathrm{InH}$ ). Ces 24 énoncés ont été enregistrés dans une pièce isolée du bruit, par une locutrice native du français, âgée d'une quarantaine d'années, originaire de la région parisienne et vivant en Isère depuis une quinzaine d'années.

Dans chaque bloc de 6 énoncés, 3 types de variables sociolinguistiques sont concernées : deux variables syntaxiques, deux variables phonétiques, et deux variables discursives : 


\begin{tabular}{|c|c|}
\hline \multirow[t]{2}{*}{ Variables syntaxiques } & « ne » de négation réalisé/non réalisé \\
\hline & relative avec « dont »/avec « que » \\
\hline \multirow[t]{2}{*}{ Variables phonétiques } & liaison facultative réalisée/non réalisée \\
\hline & /1/ dans le pronom clitique « il » réalisé/non réalisé \\
\hline Variables discursives & $\begin{array}{l}\text { particules discursives plutôt « surveillées » (ex : euh non) ou plutôt } \\
\text { "familières » (ex : oh mais) }\end{array}$ \\
\hline
\end{tabular}

Tableau 1 : variables sociolinguistiques utilisées dans la tâche de répétition

Lors de cette tâche, les sujets doivent répéter soit un énoncé stylistiquement homogène (exemples 1 cidessous), soit un énoncé non homogène (exemples 2) :

Ex1 :

Formel Homogène $(\mathrm{FH})$ : C'esT un (avec liaison facultative réalisée) pays accueillant où il ferait certes bon vivre.

Informel Homogène $(\mathrm{IH}): \mathrm{Tu}$ (absence du «ne » de négation) vas pas mett(re) tes pompes dégueu dans ma caisse, nan?

Ex2:

Formel non Homogène $(\mathrm{FnH})$ : Certes les débordements dans l'hémicycle étaient (sans liaison) inévitables. (énoncé globalement formel avec une variante incohérente - ici phonétique -, l'absence de liaison facultative)

Informel non Homogène (InH) : Ouais bein $1(\mathrm{e})$ gars i(l) n'avait pas franch(e)ment $1(\mathrm{e})$ choix au final. (énoncé globalement informel avec une variante incohérente - ici syntaxique -, la présence du ne de négation)

Entre l'audition du modèle et la répétition, il a été demandé aux sujets de lire un nombre (délai de répétition de 3 secondes à partir de la fin de l'énoncé entendu), afin de différer légèrement la reproduction de l'énoncé qui doit ainsi être stocké en mémoire de travail avant d'être restitué et éventuellement reconstitué à partir des représentations stylistiques dont nous voulons tester l'action. Les énoncés ont été proposés aux sujets dans un ordre aléatoire géré par le logiciel E-prime.

Le caractère globalement formel ou informel des énoncés, qu'ils soient homogènes ou non-homogènes, a été validée lors d'une tâche de jugement soumise à 62 étudiants de Licence de Sciences du Langage. Pour chaque énoncé entendu, les étudiants devaient déterminer s'ilavait été produit plutôt dans une situation où la locutrice enregistrée surveillait son langage ou plutôt dans une situation où elle ne surveillait pas son langage. Ils devaient également préciser leur degré de certitude sur une échelle allant de 0 (pas sûr du tout) à 4 (tout à fait sûr) ( $c f$. annexe 2). Les énoncés que nous avions conçus comme informels sont jugés informels par $85 \%$ des juges et les énoncés informels par $95 \%$ d'entre eux ${ }^{2}$. Les taux de certitude sont bons : 3,23/4 pour les énoncés formels et 3,73/4 pour les énoncés informels. Cette vérification de la catégorisation «intuitive » globale des énoncés indique qu'ils sont globalement bien catégorisés soit en formel soit en informel, en cohérence avec la conception du test.

Les énoncés répétés par les 58 adultes participant à l'expérience ont été transcrits par deux sociolinguistes. Les cas de désaccord ont été discutés puis résolus par des décisions communes. Ont été considérées comme des non réponses, l'absence totale de répétition, ou encore l'absence de répétition du segment contenant la variable sociolinguistique cible. Les traitements statistiques ont été effectués à partir des pourcentages individuels de variantes modifiées (du formel au non formel ou dans le sens inverse) calculés sur le nombre total de réponses. Nous avons effectué une analyse de variance à mesures répétées incluant trois facteurs : l'homogénéité de l'énoncé (homogène / non homogène), la formalité de l'énoncé (formel / informel), le niveau linguistique de la variante cible (syntaxique / phonologique / discursif). 


\section{Résultats}

Nos prédictions concernant cette tâche étaient les suivantes :

- à l'écoute d'un énoncé homogène, la répétition de la variante cible est identique à l'input ;

- à l'écoute d'un énoncé non homogène, il y a restauration de la variante cible en cohérence avec l'ensemble du message, donc répétition non identique à l'input, par exemple la variante informelle entendue est produite dans sa version formelle si l'énoncé est globalement formel.

Ex :

$>$ Input : Certes les débordements dans l'hémicycle étaient (sans liaison) inévitables.

> Répétition : Certes les débordements dans l'hémicycle étaienT (avec liaison) inévitables.

Afin de tester ces prédictions, nous avons cherché à savoir si les sujets modifiaient significativement plus les énoncés stylistiquement non homogènes (en restaurant la variable congruente avec le reste de l'énoncé) que les énoncés stylistiquement homogènes. Ces derniers jouent le rôle d'une condition contrôle, qui permet de s'assurer que la restauration résulte bien de la contradiction entre la variante et le reste de l'énoncé, et pas du simple fonctionnement de la mémoire ou de la tâche de répétition. Notamment cette condition contrôle permet de vérifier que la modification des variantes n'est pas due uniquement à une surveillance ou une auto-correction systématique vers le style formel, qui pourraient être induites par la situation expérimentale.

Les analyses statistiques menées sur la base de la transcription des énoncés répétés indiquent généralement que les effets simples des trois facteurs, leurs interactions deux à deux et l'interaction à trois facteurs sont significatifs $\left(F>8, p \leq 001, \eta^{2}>0.233\right)$.

Plus précisément, les sujets modifient davantage les énoncés stylistiquement non homogènes (en restaurant la variable congruente avec le reste de l'énoncé) que les énoncés stylistiquement homogènes $\left(\mathrm{F}(1,56)=351, \mathrm{p}<0.001, \eta^{2}=0.862\right)$, comme l'illustre la figure 2. Les répétitions différentes sont très fréquentes dans le cas des énoncés stylistiquement non homogènes $(44.1 \%)$, et très peu fréquentes dans les énoncés homogènes $(8 \%)$, dont les variantes sont répétées à l'identique dans plus de $90 \%$ des cas.

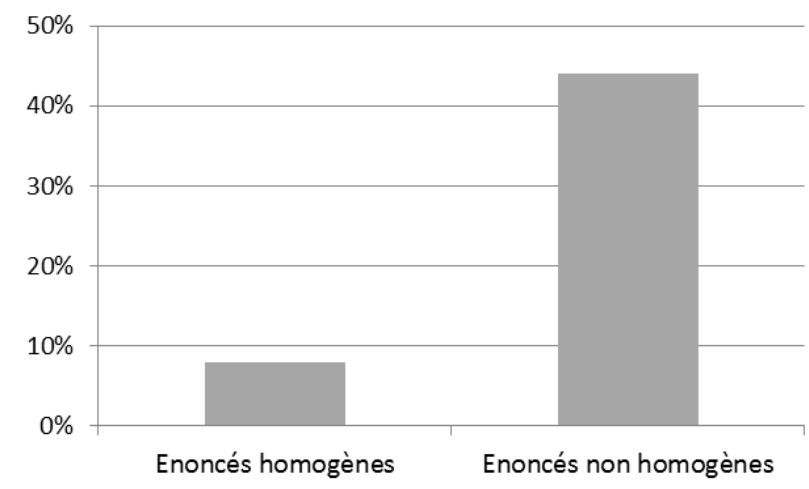

Figure 2 : répétitions non identiques au modèle entendu, en fonction du critère d'homogénéité stylistique de l'énoncé

Les pourcentages de répétitions non identiques varient non seulement en fonction du critère d'homogénéité mais également en fonction du niveau linguistique, ces deux facteurs interagissant l'un avec l'autre $\left(\mathrm{F}(2,55)=103, \mathrm{p}<0.001, \eta^{2}=0.790\right)$. Cette interaction est représentée dans la figure $3^{3}$. En effet, aux trois niveaux linguistiques, les sujets modifient davantage les énoncés stylistiquement non homogènes (en restaurant la variable congruente avec le reste de l'énoncé) que les énoncés stylistiquement homogènes. Toutefois, on constate sur le graphe que la différence avec la condition contrôle est beaucoup plus importante aux niveaux syntaxique et phonétique qu'au niveau discursif. 


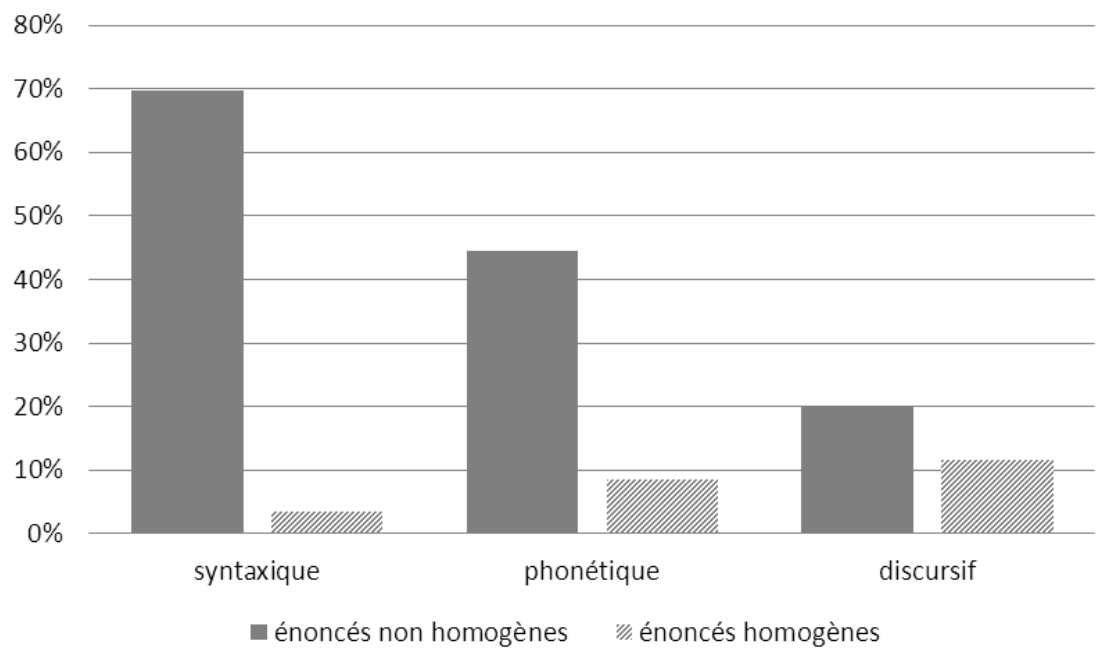

Figure 3 : répétitions non identiques au modèle entendu, en fonction du critère d'homogénéité stylistique de l'énoncé et du niveau linguistique de la variable cible.

L'interaction à trois variables étant significative $\left(F(2,55)=8, p=0.001, \eta^{2}=0.233\right)$, nous avons approfondi cette analyse en comparant les énoncés homogènes et non homogènes par niveau linguistique en fonction de la formalité,

Pour illustrer cette comparaison, prenons par exemple la variable sociolinguistique ne de négation, présente dans les énoncés ci-dessous :

$\mathrm{FH}$ : Nous ne connaissions même pas le président $\mathrm{d}(\mathrm{e})$ cette association.

FnH : C'esT un prix symbolique même si le gouvernement (ne) veut pas parler de gratuité

$\mathrm{IH}: \mathrm{Tu}(\mathbf{n e})$ vas pas mett(re) tes pompes dégueu dans ma caisse, nan ?

InH : Ouais bein le gars i(l) n'avait pas franch(e)ment l(e) choix au final.

Cette nouvelle analyse permet de répondre à deux questions :

1/ Un ne absent dans un énoncé formel non homogène comme FnH est-il plus souvent modifié (et donc restauré) qu'un ne absent dans un énoncé informel homogène comme IH ?

2/ Un ne présent dans un énoncé informel non homogène comme InH est-il plus souvent modifié (et donc supprimé) qu'un ne présent dans un énoncé formel homogène comme FH ?

Une réponse positive à ces deux questions pour chaque niveau linguistique permettrait de conclure que le phénomène de RS se maintient quelle que soit la nature linguistique de la variable sociolinguistique d'une part, et quelle que soit la direction de la restauration d'autre part: soit le remplacement d'une variante informelle par une variante formelle, soit le remplacement inverse.

Nous avons donc effectué des comparaisons par paires pour chaque niveau linguistique, en opposant FnH et IH d'une part, et InH et FH d'autre part, comme le montre le tableau 2 ci-après. 


\begin{tabular}{|c|c|c|c|c|c|c|}
\hline & FnH & IH & Test $\mathrm{t}$ & InH & FH & Test t \\
\hline syntaxique & $89,5 \%$ & $4,4 \%$ & $\mathrm{t}=22,706, \mathrm{p}<0,0001$ & $54,3 \%$ & $2,6 \%$ & $\mathrm{t}=11,681, \mathrm{p}<0,0001$ \\
\hline phonétique & $66,4 \%$ & $11,2 \%$ & $\mathrm{t}=10,982, \mathrm{p}<0,0001$ & $24,1 \%$ & $6,0 \%$ & $\mathrm{t}=4,501, \mathrm{p}<0,0001$ \\
\hline discursif & $19,8 \%$ & $16,4 \%$ & $\mathrm{t}=0,814, \mathrm{p}=0,419$ & $54,3 \%$ & $2,6 \%$ & $\mathrm{t}=3,401, \mathrm{p}=0,001$ \\
\hline
\end{tabular}

Tableau 2 : comparaison des énoncés homogènes et non homogènes selon la formalité de l'énoncé par niveau linguistique

Dans cinq cas sur les six comparaisons figurant dans ce tableau, les sujets modifient plus souvent la variante cible dans la condition non homogène qu'ils ne la modifient dans la condition homogène correspondante. La seule comparaison non significative concerne le niveau discursif lorsque la restauration consiste à remplacer un marqueur discursif informel par un marqueur discursif formel (cases grisées dans le tableau). En effet, le faible taux de RS des variantes discursives dans les énoncés formels non homogènes $(19,8 \%)$ n'est pas significativement différent des répétitions modifiées après l'écoute d'énoncés informels homogène contenant des variantes de ce type $(16,4 \%)$.

Les énoncés concernés sont les suivants :

FnH5. Nous pouvons sans doute nous y soustraire hein.

IH5. Écoute $\mathrm{t}(\mathrm{u})$ est vachement sympa merci hein.

FnH6. Oh mais aujourd'hui les gens ne se marient plus à 25 ans à moins d'y être contraints.

IH6. Oh mais lui pour s'en remett(re) franch(e)ment ça été chaud.

Dans ces deux cas, FnH n'induit pas plus de modification que IH, ce qui était pourtant attendu : les variantes choisies étant considérées comme informelles, l'hypothèse était que les sujets auraient tendance à les supprimer dans les énoncés formels : suppression de hein considéré comme un marqueur discursif informel, dans FnH5 et suppression de oh mais également considéré comme un marqueur discursif formel, dans FnH6.

Malgré ce dernier résultat, cette expérience met en évidence des manifestations non incidentes et massives (voir les forces d'effet évaluée par $\eta^{2}$ ) du phénomène de restauration stylistique. L'existence de ce phénomène suggère que le locuteur construit des représentations cognitives homogènes des styles. Autrement dit, il catégoriserait les énoncés entendus en variétés discrètes homogènes, indépendamment de la présence d'éléments dissonants dans ces énoncés.

Néanmoins, les écarts entre les différents types de variables et entre les contextes formels et informels nécessitent des analyses complémentaires. En effet, comment expliquer que certaines variantes soient quasiment systématiquement restaurées, alors que d'autres ne le sont pas ? Une première hypothèse que nous avançons concerne la saillance perceptive des variantes. En effet, les taux de restauration les plus faibles concernent les variables discursives, et particulièrement les énoncés cités ci-dessus. Nous pouvons faire l'hypothèse que les variables cibles sont ici suffisamment saillantes perceptivement (notamment du fait de la place dans l'énoncé, en tout début ou toute fin de phrase, et de la courbe prosodique) pour que les sujets les «entendent», les retiennent à court terme et les restituent à l'identique, malgré la non congruence supposée avec le reste de l'énoncé. Les marqueurs de discours, comme les éléments lexicaux, pourraient en effet être plus facilement perçus que des variables phonologiques par exemple.

Une autre piste d'explication concerne le caractère intuitif et exploratoire de la nature formelle / non formelle de ces variables. En effet, si les études sociolinguistiques ont largement démontré l'association ne de négation/contexte formel (Armstrong, 2002) ou encore liaison facultative/contexte formel (Lucci, 1983; Moisset, 2000), les enquêtes manquent pour déterminer objectivement quelles seraient les marqueurs de discours plutôt formels $v s$ plutôt informels. 
Une troisième piste d'explication, notamment pour le cas de l'énoncé FnH6, pourrait être la plus faible capacité de certains contextes à activer le schéma "formel". En effet, dans la tâche de jugement, l'énoncé en question est catégorisé comme "formel" par moins de $60 \%$ des sujets. Ce flottement explique sans doute en partie le caractère moins systématique et plus fluctuant des résultats que nous avons recueillis.

\section{Conclusion et prolongements}

Ces premières analyses permettent donc de conclure à l'existence du phénomène de RS, et conséquemment à un processus de catégorisation cognitive de l'hétérogène stylistique en variétés homogènes. Si la variation stylistique dans les productions authentiques des locuteurs ne peut être réduite à des registres de langue finis et dénombrables, la perception de cette variation semble pourtant se rapprocher de ce processus de «gommage » recréant de l'homogène dans l'hétérogène et ignorant les dissonances stylistiques. La tâche de répétition utilisée ne permet toutefois pas de décider si le phénomène que nous mettons ici pour la première fois en évidence se situe au niveau de la perception ou de la production. En effet, on peut estimer que les schémas stylistiques homogènes activés par la tonalité globale des énoncés agissent au niveau de leur perception. Dans ce cas, les sujets ne percevraient pas les variantes sociolinguistiques en incohérence avec le reste de l'énoncé. Mais on peut supposer également que ces schémas agissent après la perception des énoncés, lors des quelques secondes précédant leur production. Dans ce cas, ce serait lors de la reconstruction de l'énoncé dans la mémoire de travail en vue de sa production que les variantes seraient modifiées en fonction du contexte global. D'autres expériences sont nécessaires pour trancher sur ce point.

La réflexion en cours sur le protocole expérimental et les enjeux théoriques nécessite une collaboration scientifique plus serrée entre sociolinguistique et cognition sociale. En effet, la formation des impressions est un objet de cognition sociale qui renvoie au processus d'organisation et d'intégration des informations qui aboutit à une impression cohérente, unifiée et située d'autrui (Ritzer, 2007). Cet objet est analogue à celui de la cognition sociolinguistique qui examine la récupération d'informations indexicales lors du traitement des variations. Une autre notion importante est celle d'automaticité de la vie sociale (Bargh \& Williams, 2006). La perception non consciente de traits à valeur sociale dans l'environnement active automatiquement des stéréotypes qui influencent nos comportements (Bargh et al., 1996). Cette automaticité doit être prise en compte dans la cognition sociolinguistique ainsi que les méthodes pour l'explorer (e.g. priming ou amorçage social). Soulignons que cette rencontre entre cognition sociale et sociolinguistique a été présentée comme une nécessité par certains auteurs (Campbell-Kibler, 2010), mais qu'elle a été rarement mise en œuvre à ce jour.

L'expérimentation en cours, qui fait suite à cette première étude dont nous avons ici décrit les résultats, se nourrit d'une collaboration entre les deux disciplines ${ }^{4}$. Elle explore la question des variations sociales potentielles entre les sujets, en intégrant notamment des variables sociolinguistiques liées à leurs parcours de socialisation, telles que la mixité du réseau social. L'une des questions qui nous intéresse est de savoir si le fait d'être davantage familier d'un style (formel ou informel) modifie ou non les taux de RS. Par ailleurs, nous vérifierons que l'activation du stéréotype agit sur les taux de RS, en recourant à la technique de l'amorçage social, largement utilisée en psychologie cognitive. Les analyses à venir devraient ainsi permettre de mettre en évidence d'éventuelles différences dans les processus de catégorisation des styles en fonction du profil social des locuteurs. 


\section{Références bibliographiques}

Armstrong, N. (2002). Variable deletion of French ne: a cross-stylistic perspective. Language Sciences 24 (2), 153173.

Bargh, J.A., Chen, M. \& Burrows, L. (1996). Automaticity of social behavior: direct effects of trait construct and stereotype activation on action. Journal of personality and social psychology, 77 (2), 230-244.

Bargh, J.A., \& Williams, L.E. (2006). The automaticity of social life, Current Directions in Psychological Science, $15,1-4$.

Buson, L. (2010). La compétence métapragmatique chez les enfants de 9 à 11 ans. Processus cognitifs et effets de saillance. Actes du Congrès Mondial de Linguistique Française. Nouvelle Orléans (États-unis). Juillet 2010.

Buson, L. \& Billiez, J. (2013). Representations of Stylistic Variation in 9-11 year-olds. Cognitive Processes and Salience. Linguistics, 51(2), 325-354.

Campbell-Kibler, K. (2010). New directions in sociolinguistic cognition. sociolinguistic cognition, University of Pennsylvania Working Papers in Linguistics, 15(2), 31-39.

Chevrot J.P. \& Foulkes, P. (Eds) (2013). Language Acquisition and Sociolinguistic Variation, special issue of Linguistics.

Coupland, N. (2007). Style. Language variation and identity. Cambridge University Press.

De Jong, D. (1994). La sociophonologie de la liaison orléanaise. In Lyche, C. (dir.), French Generative Phonology: retrospective and perspectives (pp. 95-130). Association for French Language Studies in association with the European Studies Research Institute, Salford,

Eckert, P. \& Rickford, J. R. (2001). Style and sociolinguistic variation. Cambridge University Press.

Gadet, F. (2007 [2003]). La variation sociale en français. Ophrys.

Hay, J. \& Drager, K. (2010) Stuffed toys and speech perception. Linguistics, 48(4):865-892.

Hay, J., Nolan, A. \& Drager, K. (2006a). From fush to feesh: Exemplar priming in speech perception, The Linguistic Review, 23, 351-379.

Irvine, J. (2001). "Style" as distinctiveness: the culture and ideology of linguistic differentiation. In Eckert, P. \& Rickford, J. R. (Eds.), Style and sociolinguistic variation (pp.21-43). Cambridge: Cambridge University Press.

Johnson, K., Strand, E.A. \& D'Imperio, M. (1999). Auditory-visual integration of talker gender in vowel perception, Journal of Phonetics. 27, 359-384.

Labov, W., (1972). Sociolinguistic Patterns. Blackwell, Oxford.

Lafontaine, D. (1986). Le parti pris des mots. Mardaga, Bruxelles.

Lahire, Bernard \& Rosental, Claude (2008). Introduction. In Lahire, B. \& Rosental, C. (dir.), La cognition au prisme des sciences sociales, Paris, Editions des Archives Contemporaines, 7-19

Landragin, F. (2004). Saillance physique et saillance cognitive. CORELA, 2, (2), http://corela.edel.univpoitiers.fr/document.php?id=142. Consulté le 3 juin 2009.

Landragin, F. (2005). Modélisation de la saillance visuelle et linguistique. Sixième Colloque des Jeunes Chercheurs en Sciences Cognitives (CJCSC'05) (pp.157-162). Bordeaux.

Loudermilk, Brandon (2013). Psycholinguistic approaches. In Bayley, R. Cameron, R. \& Lucas, C. (Eds), The Oxford Handbook of Sociolinguistics. Oxford: Oxford University Press, 132-152.

Lucci, V. (1983). Étude phonétique du français contemporain à travers la variation situationnelle. Grenoble: Publications de l'Université des Langues et Lettres de Grenoble.

Moisset, C. (2000). Variable liaison in Parisian French. Ph.D. dissertation, University of Pennsylvania, Philadelphia.

Moreau, M.-L. \& Brichard, H. (1997). La part de la subjectivité dans l'évaluation de la qualité linguistique : une étude en Belgique francophone. Parole, 2, pp.121-145. 
Niedzielski, N. (1999). The Effect of Social Information on the Perception of Sociolinguistic Variables, Journal of Language and Social Psychology, 18: 62-85.

Ritzer, G.(ed.) (2007). Encyclopedia of sociology (11 vols.). Oxford: Blackwell.

Squires, L. M. (2011). Sociolinguistic Priming and the Perception of Agreement Variation: Testing Predictions of Exemplar-Theoretic Grammar, Ph.D. Thesis, University of Michigan.

Staum Casasanto, L. (2009). Experimental investigations of sociolinguistic knowledge. Stanford, CA: Stanford University Ph.D. Dissertation.

Trudgill, P. (1975). Sex, covert prestige and linguistic change in the urban British English of Norwich. In Thorne, B., Henley, N. (dir.), Language and Sex: Difference and Dominance (p. 88-104). Newbury House Publishers, Rowley,

Trudgill, P. (1995). Sociolinguistics: An Introduction to Language and Society, third ed. Penguin Books, London.

Warren, R. M. (1970). Perceptual restoration of missing speech sounds. Science, 167: 392-393. 


\section{Annexe 1 : énoncés soumis à la répétition}

Formels Homogènes

FH1. Nous ne connaissions même pas le président d(e) cette association.

FH2. Je m'interroge sur la façon donT ils conçoivent l'enseignement en général.

FH3. C'esT un pays accueillant où il ferait certes bon vivre.

FH4. Etant donné qu'il travaille à l'hôpital, démissionner esT une décision délicate.

FH5. De plus, euh non, certains articles évoquaient plutôt une restructuration.

FH6. Oui bon, la situation est certes éminemment complexe.

Formels non Homogènes

FnH1. C'esT un prix symbolique même si le gouvernement veut pas parler de gratuité.

FnH2. L'écueil majeur concerne la manière qu'on enseigne l'informatique à l'école.

FnH3. Certes les débordements dans l'hémicycle étaient inévitables.

FnH4. Pour subvenir à ses besoins, bien entendu, i(l) faut d'autres sources de revenus.

FnH5. Nous pouvons sans doute nous y soustraire hein.

FnH6. Oh mais aujourd'hui les gens ne se marient plus à 25 ans à moins d'y être contraints.

Informels Homogènes

IH1. Tu vas pas mett(re) tes pompes dégueu dans ma caisse, nan ?

IH2. Nan c(e) que j(e) te parlais t(ou)t à l'heure, c'(é)tait un aut(re) plan foireux.

IH3. C'est un appart avec un pote (il) y a pas la télé.

IH4. Alors i(I) regarde le truc et i(l) dit oh mais c'est quoi ça ?!

IH5. Écoute $\mathrm{t}(\mathrm{u})$ est vachement sympa merci hein.

IH6. Oh mais lui pour s'en remett(re) franch(e)ment ça été chaud.

Informels non Homogènes

InH1. Ouais bein le gars i(l) n'avait pas franch(e)ment l(e) choix au final.

$\mathrm{InH} 2$. Essaye de pas $\mathrm{m}(\mathrm{e})$ refiler $1(\mathrm{e})$ matos pourri dont $\mathrm{t}(\mathrm{u})$ as parlé l'aut(re) fois.

InH3. C'esT un sale gosse j(e) te jure il est grave.

InH4. Ouais il bosse tout $1(\mathrm{e})$ temps c'est abusé.

InH5. Euh non en vrai j'avais bien les boules de pas capter ses questions.

InH6. Oui bon en même temps comment i(l) s(e) galère avec sa meuf. 


\section{Annexe 2 : type de consigne fournie aux sujets pour la tâche de jugement des énoncés}

\section{Consigne}

Nous allons vous faire écouter 24 énoncés produits par la même femme. Nous vous demandons de les écouter attentivement puis en cochant une case, de juger si elle les a produits :

$\square$ plutôt dans une situation où elle surveille son langage

$\square$ plutôt dans une situation où elle ne surveille pas son langage

Dans un deuxième temps, nous vous demandons d'estimer le degré de certitude de votre jugement en entourant un chiffre sur échelle qui va de 0 à 4 allant de pas sûr du tout à tout à fait sûr.

Dans quelle mesure êtes-vous sûr de votre jugement?

Pas sûr du tout

0
1
2
Tout à fait sûr 4

\footnotetext{
${ }^{1}$ Le terme de restauration stylistique fait référence au phénomène de restauration phonémique avancé depuis plusieurs décennies pour étayer les aspects descendants de la perception de la parole (Warren, 1970).

${ }^{2}$ En ce qui concerne les énoncés informels, on remarque qu'ils sont massivement jugés informels par les étudiants (entre 92 et $100 \%$ des sujets selon les énoncés). Pour les énoncés formels, nous constatons davantage de variation dans les jugements avec une étendue de $53 \%$ à $98 \%$ d'individus en faveur de la formalité selon l'énoncé en question.

${ }^{3}$ Les taux de la figure 3 sont calculés sans différencier les énoncés formels et informels.

${ }^{4}$ Projet financé par le Pôle Grenoble Cognition, associant le LIDILEM (laboratoire de linguistique et de didactique des langues) et le LIP (laboratoire interuniversitaire de psychologie).
} 